\title{
DETECTION OF BIODEGRADATION DEGREE OF SLUDGE USING DIELECTRIC MEASUREMENT
}

\author{
SÁndor Beszédes, Katalin PAPP-Sziládi, GÁbor KeszThelyi-Szabó, Cecília \\ HodúR
}

\author{
University of Szeged Faculty of Engineering \\ Institute of Process Engineering \\ Moszkvai krt. 9, H-6725 Szeged, Hungary \\ beszedes@mk.u-szeged.hu
}

\begin{abstract}
Pre-treatments applied before biological sludge utilization technologies aim to modify the sludge structure for enhanced disintegration degree and biodegradability. Among the thermal pre-treatments methods, microwave irradiation is suitable to degrade the polymeric structure of sludge, and to increase the solubility of organic matters. Energetic efficiency of microwave heating is mainly determined by the dielectric properties, such as dielectric constant and dielectric loss factor. Dielectric properties are influenced by the frequency, temperature; composition and consistency of irradiated material, state and bond of water etc. Therefore, physicochemical changes of sludge structure; e.g. hydrolysis of macromolecules, degradation of cell wall of microorganisms, aggregation of particles; contribute to the change of dielectric parameters, as well. In our work we investigated the correlation between the dielectric parameters and structural change and biodegradability indicators. In the case of municipal wastewater, the change of organic matter removal efficiency during wastewater purification technology at a wastewater treatment plant can be detected by the change of dielectric constant. Results related to sludge processing show, that change of organic matter solubility and aerobic biodegradability correlate the change of dielectric loss factor and dielectric constant. With the degradation of polymeric structure of sludge matrix and decomposition of macromolecules caused by thermal effects or chemical pre-treatments led to increased mobility of ions and enhanced polarization of molecules. These effects led to increased dielectric constant and loss factor, what make possible to preindicate the efficiency of sludge pre-treatment processes by an in line and real time measurement method.
\end{abstract}

Keywords: microwave, dielectric properties, biodegradability, sludge, wastewater)

\section{INTRODUCTION}

Attention to microwave heating applications is continuously growing, because of the special mechanisms of microwave irradiation for energy transfer. On the contrary of the conventional heating mechanisms, energy is delivered directly into the materials during microwave heating due to the molecular level interactions with high frequency electromagnetic field. Because of the high energy density and volumetric heating effect, microwave irradiation is suitable to achieve very short process time, and, in some cases, microwave irradiation induces special structural properties or new way for chemical reactions (KAPPE, 2004). The behavior of materials in electromagnetic field can be characterized by the complex permittivity. The complex permittivity has real part, named dielectric constant and an imaginary part, named as loss factor. Dielectric constant measures the ability of material to store the external electric field; the loss factor shows the energy absorption capability of materials. Heat generation efficiency of microwave irradiation can be given by the power dissipation which can be calculated from the strength of electric field, the frequency and the dielectric parameters, respectively (BRODIE ET AL., 2014).

Depending on the physicochemical structure and composition of processed material, and the applied frequency range, heating mechanisms of microwave irradiation is occurred by dielectric polarization and/or ionic conduction. Orientation polarization or diploes rotati on 
occurs due to the reorientation of permanent dipoles in oscillating electromagnetic field (BARBA AND D'AMORE, 2012). The materials containing free electrons charge polarization can be observed caused by the modification of position of electrons, resulted in nonuniform distribution of charges. In the industrially scale used microwave frequency ranges, and mainly for high water contented materials the dipole rotation is the dominant mechanisms, but ionic dissipation phenomena can be also occurred, if ions are presented in the irradiated materials (BRODIE ET AL., 2014).

In complex structured materials both mechanisms can be determinative at the same frequency, for example if the state of water content is changed. If thermal or mechanical stress was applied cell walls can disrupt, therefore the intracellular components are released increasing the free water content. The dielectric loss factor decrease with temperature until the effects of dipoles dominates, but then start to increase due the present of ionic compounds originated from intracellular liquor. There can be found process in which ionic components are dosed to the wastewater to increase the heating efficiency of microwave irradiation. Ionic conduction can be dominant, if the ionic compounds have migration ability, for example in solutions or suspensions. If the dipolar rotation mechanisms dominated over the ionic dissipation, decreasing of dielectric property values was observed, because temperature increment increases the molecular disorder. On the other hand, due to the reduced dielectric loss factor, the penetration depth of electromagnetic waves increases (HOLTZE ET AL., 2006). When the penetration depth is lower than the half of the characteristic dimension of irradiated sample uniform heating cannot be achieved. But heat can be transferred from the heated skin layer towards the core of material by conduction or convection mechanisms. The effects of microwaves are divided into thermal and athermal effects. Thermal effects are occurred by the heat generation in material due to the absorption of microwave energy. Athermal effects mean the change of chemical, biochemical and physicochemical behavior of the materials caused by electromagnetic field without temperature change. The results related to the investigation of athermal effects are contradiction. It can be noticed, that activation energy of microwave irradiation in $\mathrm{GHz}$ frequency range is too low to disrupt the covalent bonds or hydrogen bonds (KAPPE, 2004).

Dielectric parameters of wastewater and sludge are not investigated in details, and there is not examined the possible relationship between the biodegradability indicators and dielectric parameters. Therefore our work focused on to determine the temperature dependency of dielectric constant of wastewater and sludge originated from municipal wastewater purification technology and food industry, respectively. Another task of research work is to investigate the correlation between the change of biodegradability and dielectric parameters.

\section{MATERIAL AND METHOD}

Municipal wastewater samples were originated from a local wastewater treatment plant, purified wastewater was sampled after biological stage of WWTP. Raw (untreated) food industry wastewater sludge sampled from the puffer tank of wastewater line of a dairy factory.

MW pre-treatments were performed in a tailor-made continuously flow microwave reactor; equipped by a continuously irradiating variable power magnetron operating at a frequency of $2450 \mathrm{MHz}$. Power of magnetron was adjusted by the heating voltage. The flow rate was varied by the speed of peristaltic pump. Dielectric constant $\left(\varepsilon^{\prime}\right)$ was measured in a tailor made dielectrometer system equipped with a dual channel power meter (Rohde \& 
Schwarz). $\varepsilon$ ' was calculated from the phase shift $(\phi)$ and reflection coefficient $(\Gamma)$.

Chemical oxygen demand (COD) was measured by colorimetric method (APHA, 2005). Biodegradable fraction of organic matters was quantified by 5 days biochemical oxygen demand (BOD) tests. Aerobic biodegradability was characterized by the ratio of BOD to COD

\section{RESULTS}

In the first series of experiments, the effect of continuously flow microwave pre-treatment on aerobic biodegradability of dairy processing sludge was investigated. Our results show that microwave power (MWP) and volumetric flow rate has also significant effect on biodegradability $(\alpha=5 \%)$. Microwave pre-treatment has been verified suitable to increase the biodegradability of dairy sludge. But after a certain value, increasing of MWP resulted in lower biodegradability. Optimum range for MWP and flow rate was determined as 400$500 \mathrm{~W}$, and 20-45 $\mathrm{Lh}^{-1}$, respectively (Figure 1).

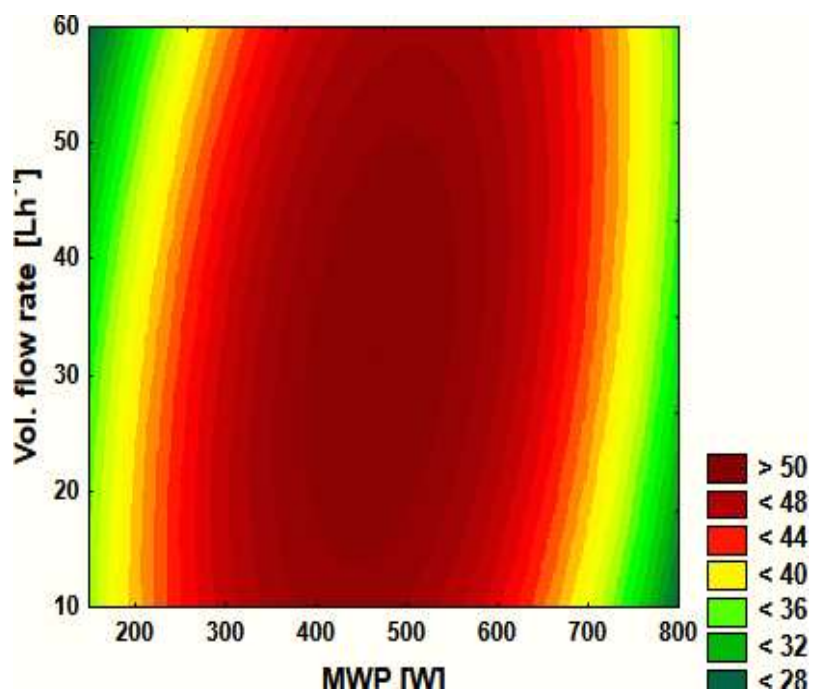

a)

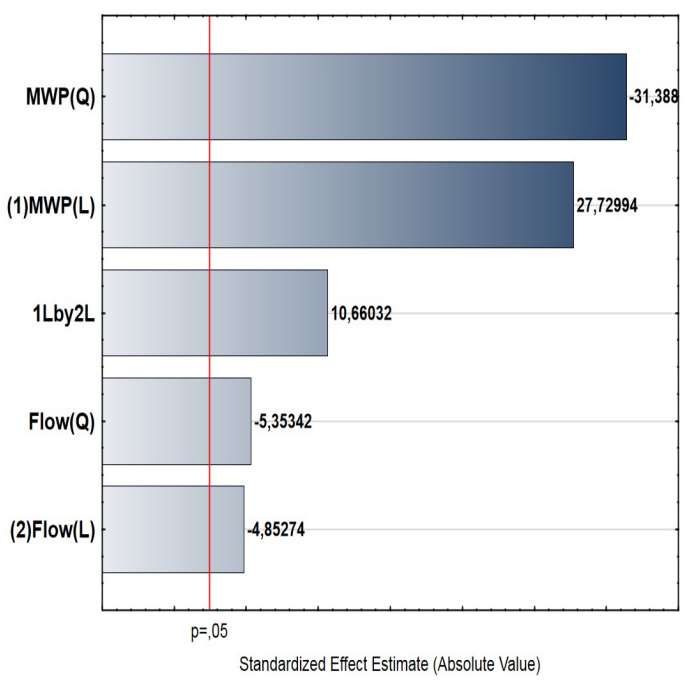

b)

Figure 1. Contour plot (a) and Pareto chart (b) for the change of biodegradability of dairy sludge

Similar tendency has been arisen for the change dielectric constant as the function of MWP and flow rate, than that of obtained for biodegradability. During the microwave pretreatment the sludge particles were disintegrated, the solubility of organic matter content increased, furthermore the cell walls are disrupted, these effects led to enhanced biodegradability. The physiochemical changes occurred by microwave irradiation have effect on the polarization ability of different components. Due to the thermal hydrolysis of macromolecules and the release of intracellular polar and ionic compounds to sludge liquor increase the dielectric constant. After a critical thermal stress Maillard reaction and aggregation of hydrolysed organic compounds take place during the energy intensive microwave irradiation what led to decreasing in biodegradability and dielectric constant, as well (Figure 2). Considering the results of ANOVA can be established, that flow rate of pre-treatment and microwave power has also significant effect on dielectric constant, but interaction between the two parameters was not significant at confidence level of $95 \%$. 


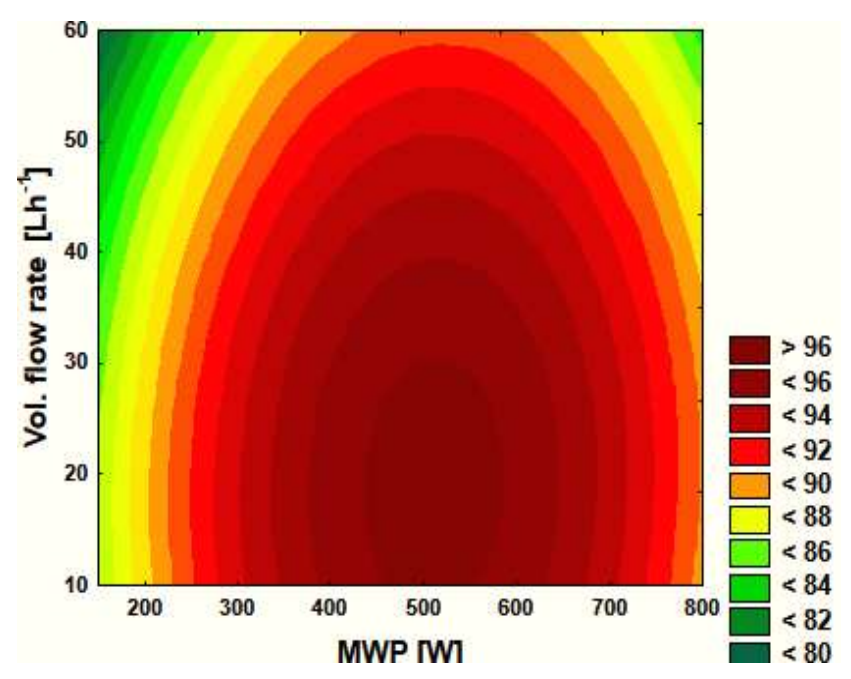

a)

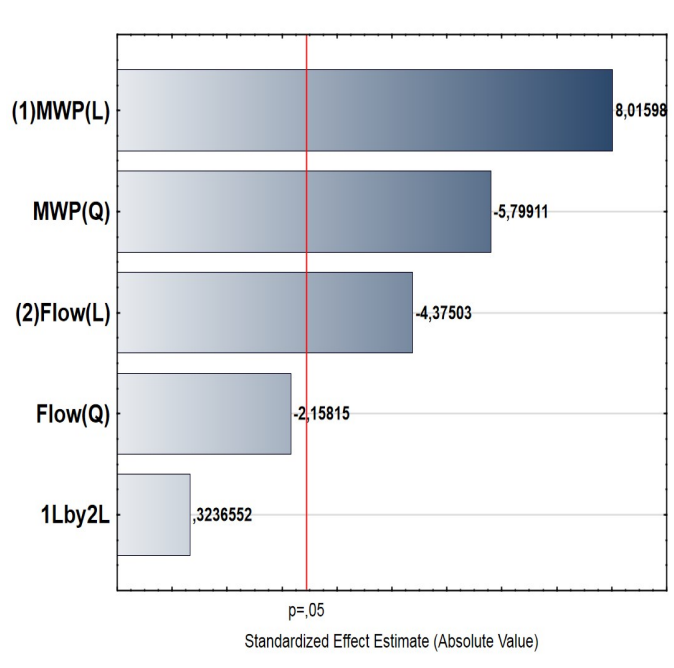

b)

Figure 2. Contour plot (a) and Pareto chart (b) for the change of dielectric constant (dielectric constant was measured at $25^{\circ} \mathrm{C}$ for all samples)

To further investigate the applicability of dielectric measurement for sludge and wastewater treatment purposes, municipal wastewater has been involved into the research work. Figure 3 show the temperature dependency of dielectric constant for raw (fresh) municipal wastewater (b) and purified wastewater (a) at different flow rate ranges. Purified wastewater samples are originated from purification technology in which mechanical and biological treatment stage was applied.

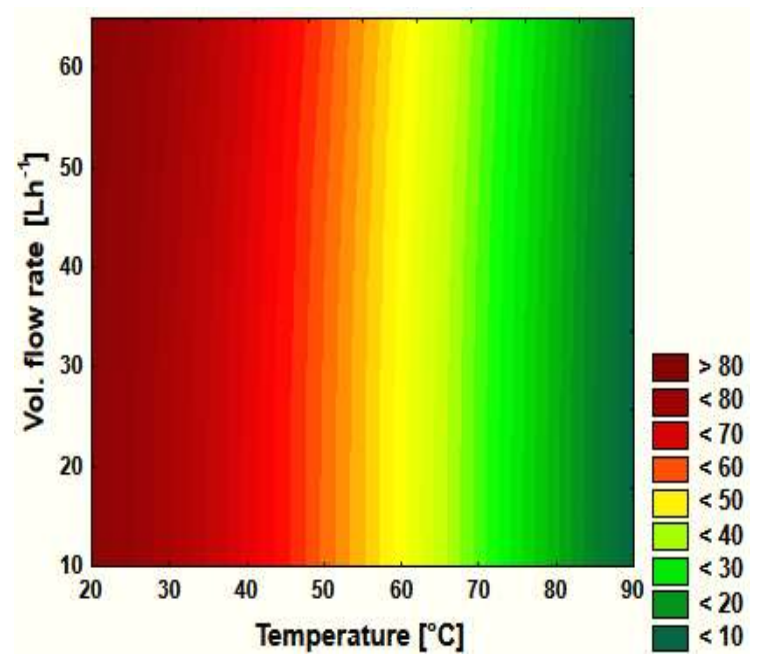

a)

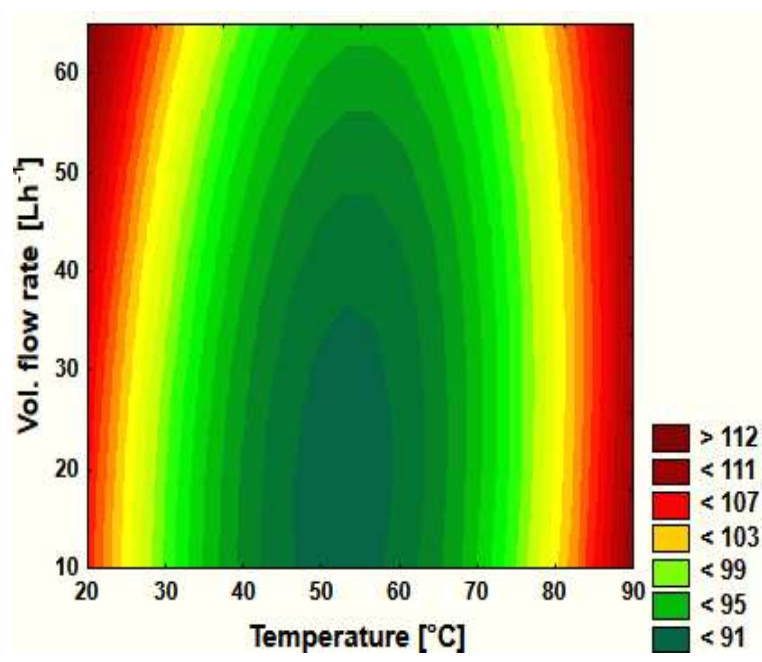

b)

Figure 3. Dielectric constant of purified (a) and raw (b) municipal wastewater as function of temperature and flow rate

The tendency of dielectric behaviour of purified wastewater was similar that of obtained for pure water, i.e. dielectric constant decrease with increasing temperature, furthermore the flow rate has not significant effect on dielectric parameter. In the case of raw wastewater a decreasing tendency of dielectric constant was found in the temperature range of $15-50^{\circ} \mathrm{C}$, but temperature increment in the range of $60-90^{\circ} \mathrm{C}$ led to increasing of dielectric constant. Because the raw wastewater has higher organic matter content (COD: $954 \mathrm{mgL}^{-1}$ ) than purified water (COD $110 \mathrm{mgL}^{-1}$ ) the temperature increment led to the 


\section{2}

Review on Agriculture and Rural Development 2017 vol. 6 (1-2) ISSN 2063-4803

disintegration and hydrolysis of particulate organic matters. The difference between the absolute value of dielectric constant of purified and raw wastewater is due to the different composition. At lower temperature range the dipolar rotation mechanism dominate resulted in decreasing tendency of dielectric constant, but over a critical temperature range (50$60^{\circ} \mathrm{C}$ ), where disintegration has been taken and concentration of lower molecular weighted and polar components start to increase, the tendency of change in dielectric constant turn to increase (Figure 3.b). At higher temperature range the ionic compounds of intracellular substance have been released, therefore the ionic conduction mechanisms contribute to the increment of dielectric constant, as well. In the case of high organic matter contented raw wastewater the change of flow rate during irradiation has also effect on the instantaneous value of dielectric constant, because the change of velocity affect the disintegration degree of organic particles, and migration ability of ions and movement of dipolar components in oscillating electromagnetic field.

\section{CONCLUSIONS}

The main aim of our research work was to examine the applicability of dielectric measurements for determination of structural change of wastewater and sludge. Our results verified that microwave irradiation is suitable to enhance the biodegradability. In continuously flow microwave pre-treatments the flow rate and the microwave power can be considered as influential parameters. Considering the results of dielectric measurements and biodegradation tests relationship can be found between dielectric constant and aerobic biodegradability of sludge. Furthermore, dielectric measurement was suitable to detect the organic matter removal efficiency during the wastewater purification technology.

\section{ACKNOWLEDGEMENTS}

The authors are grateful for the financial support provided by the OTKA, project number: K115691. This project was supported by the János Bolyai Research Scholarship of the Hungarian Academy of Sciences.

\section{REFERENCES}

BARBA, A., D'AMORE, M. (2012): Relevance of dielectric properties in microwave assisted Process. In Microwave Materials Characterization. Costanzo S. (Ed.), Intech., 170 p.

Brodie, G., Destefani, R., Schneider, P.A., Airey, L., JACOB, M.V. (2014): Dielectric properties of sewage biosolids measurement and modeling. Journal of Microwave Power and Electromegnatic Energy, 48: 147-157.

Holtze, C., Sivaramakrisham, R., Antionetti, M., Tsuwi, J., Kremer, F., Kramer, K.D. (2006): The microwave absorption of emulsions containing aqueous micro and nanodroplets: a means to optimize microwave heating. Journal of Colloid and Interface Science, 302: 651-657.

KAPPE, C.O. (2004): Controlled microwave heating in modern organic synthesis. Angewandte Chemie, 43: 6250-6284. 